\title{
Metastatic Hormone and Her-2 Positive Breast Cancer: A Community Approach
}

\author{
Ida Micaily MD ${ }^{1 *}$, Lakshmi Kolandra DO ${ }^{1}$, Shahrzad Abdollahi MD and Anthony Scarpaci MD ${ }^{2}$ \\ ${ }^{1}$ Department of Internal Medicine, Abington Jefferson Health, Pennsylvania \\ ${ }^{2}$ Abington Cancer Care at the Rosenfeld Cancer Center, Abington Jefferson Health, Pennsylvania \\ Received: 鴜 June 09, 2018; Published: 觜 June 13, 2018 \\ *Corresponding author: Ida Micaily, MD, Department of Internal Medicine, Abington Jefferson Health, Pennsylvania
}

\begin{abstract}
Metastatic hormone-receptor and HER-2 positive (triple-positive) breast cancer provides a treatment dilemma for clinicians. The current guidelines offer a variety of options in the first and second line for metastatic breast cancer. A tailored treatment approach is needed for the triple-positive metastatic breast cancer population.
\end{abstract}

Objective: To trend the therapeutic treatment selections for patients with metastatic, triple-positive breast cancer at a single, community practice.

Methods: The Abington Jefferson Health Tumor Registry and electronic medical records systems were used to retrospectively identify patients with hormone and HER-2 positive metastatic disease who were treated at Abington Jefferson Health between 2010-2016.

Results: It was found that there was variability across our population in the choice of what therapy was chosen for first, second and fourth line of treatment. The majority of patients (12 out of 15) received combination therapy with trastuzumab. In the second line, 7 out 8 patients received trastuzumab as part of their treatment regimen. In the third line, all 3 patients received trastuzumab emtansine as part of their therapy regimen. For patients who were able to survive until the fourth line and beyond, several other treatments were used.

Limitations: A small sample size is the major limitation in this study.

Conclusion: Although HR+/ Her2+ (triple positive) cancer cell type represents a subset of patients with metastatic breast cancer, there is a general lack of consensus on how to best treat this patient population. More research needs to be conducted in order for community Oncologic practitioners to be able to better coordinate care for patients with triple positive, metastatic breast cancer.

\section{Introduction}

Breast cancer is the most common cancer diagnosed globally as well as the most common cancer amongst women [1]. Approximately 6-10\% of new breast cancer cases each year are initially, clinically staged as de-novo metastatic or stage IV cancer [2]. In nonmetastatic breast cancer, surgery can often be curative for localized tumors while systemic chemotherapy with or without radiation can help shrink and eradicate disease [3]. However, metastatic breast cancer often remains an incurable malignancy that poses unique challenges in treatment options due to heterogeneity in the cancer cell population within the tumor [4]. In addition to clinical staging, the American Joint Committee on Cancer recommends the collection of biomarkers from tumor tissue, these markers are; estrogen receptor status (ER), progesterone receptor status (PR) and human epidermal growth factor status (HER2) [5]. ER and PR are also known collectively as hormonal receptors (HR). When ER, PR and HER2 are positive, these tumors are called "triple-positive". Triple positive breast cancers represent roughly ten percent of 
breast cancers [6]. While there has been impressive progress in treating triple positive breast cancer, metastatic disease continues to be a difficult task for oncologists and patients as it is incurable in majority of patients. There presently remains an unmet clinical need when it comes to a standardized treatment regimen for triplepositive metastatic breast cancer.

HER2 is a tyrosine kinase transmembrane receptor that is overexpressed on the surface of certain breast tumors and plays a key role in intracellular pathways of tumor cells. Its expression has been historically associated with aggressive disease and a worse prognosis [5]. However, the benefit of having a tumor that is HER2 positive lies in the fact that there are HER2- targeted therapies. It is commonly believed that patients that are HER2 positive benefit from HER 2 directed therapy, irrespective of HR status of the malignancy [7]. It has also recently been suggested that combination of dual HER2 blockade directed therapy plus endocrine therapy improves progression free survival [8]. Therefore, patients that are triple positive should have HER2 directed therapy included as part of the first line therapy regimen. In the current NCCN guidelines, acceptable treatment plans for HER2 positive/ HR positive advanced breast cancer patients are either HER 2 agents with hormone therapy or HER 2 agents with chemotherapy [9]. Endocrine therapy is dependent on menopausal status (pre or postmenopausal) of the patient as well exposure to endocrine therapy within the last year. The decision to switch from endocrine therapy to chemotherapy or from one line of chemotherapy to an alternative chemotherapy is multifactorial and will vary patient to patient. Factors to consider in these decisions include, noting the progression of disease on initial therapy, extent of disease on presentation and ECOG performance status. Unlike all other stages of breast cancer, treatment regimens are at the discretion of the medical oncologist. Due to tumor cell heterogeneity, tumors that are triple positive provide a distinct challenge for clinicians in determining treatment [10]. There have been an increasing number of studies over recent years attempting to answer the question of how the cross talk between HER2+ and $\mathrm{HR}+$ affects choice of treatment in patients with triple positive metastatic disease. While there have been exciting developments in attempting to identify treatments plans in this specific patient population, there remains a lack of consensus on how to optimally approach and treat HER2+/HR+ patients. With this, further research is essential to better understand how to ideally treat these patients.

\section{Methods}

Investigators reviewed consultation notes for 15 eligible patients with metastatic HER2-positive and receptor-positive breast cancer who were treated at one community center between 2010-2016. HER2-positive status was confirmed centrally, by means of immunohistochemistry (with $3+$ indicating positive status) or fluorescence in situ hybridization (with an amplification ratio $\geq 2.0$ indicating positive status). Tumor hormone-receptor status was determined locally.

\section{Results}

A total of 206 patients who were treated between the years of 2010 to 2016 in one center with metastatic breast cancer were identified. A retrospective chart analysis demonstrated that 15 of these patients were triple positive. Patient characteristics included women with ages ranging between 51-79. All patients were postmenopausal at the time of diagnosis. Of the 15 patients analyzed, 6 patients had metastatic disease to the bone, 7 patients had metastases to the lung, 2 to the brain or CSF, 1 to the liver, 1 to the adrenal gland, and 1 patient metastasized to the ovary (Table 1 ). 8 of the patients had received adjuvant chemotherapy; 7 received neo-adjuvant chemotherapy.

Table 1: Demographic table indicating site of metastases.

\begin{tabular}{|c|c|}
\hline Site of Metastasis & Number of Patients ( out of 15) \\
\hline Bone & $10 / 15$ \\
\hline Lung & $7 / 15$ \\
\hline CSF & $1 / 15$ \\
\hline Adrenal & $1 / 15$ \\
\hline Ovary & $1 / 15$ \\
\hline Brain & $1 / 15$ \\
\hline Lymph Node & $1 / 15$ \\
\hline Liver & $2 / 15$ \\
\hline
\end{tabular}

Out of the 15 total patients, 13 received combination firstline therapy, while 2 patients received single-agent therapy. The clinical rationale for both patients who received single-agent chemotherapy was for better tolerability, however both patients had progressive disease with an outcome of hospice. A total of 12 out of 15 of our patients received trastuzumab as a part of their first line of chemotherapy. Of the 13 patients who received combination first line therapy, 9 patients proceeded onto second line therapy, and one patient achieved remission (Figure 1). 7 out of 8 patients received trastuzumab in their second line of therapy, and 1 out of 8 patients received single line letrozole. 5 out of 8 patients in the second line group received an aromatase inhibitor in addition to Herceptin. 3 patients went onto third line therapy; two patients receiving ado-trastuzumab emtansine, and one patient received ado-trastuzumab emtansine and lapatinib combination therapy. 2 of the 15 patients received fourth line of agents, and 1 patient was able to undergo 8 lines of therapy. 


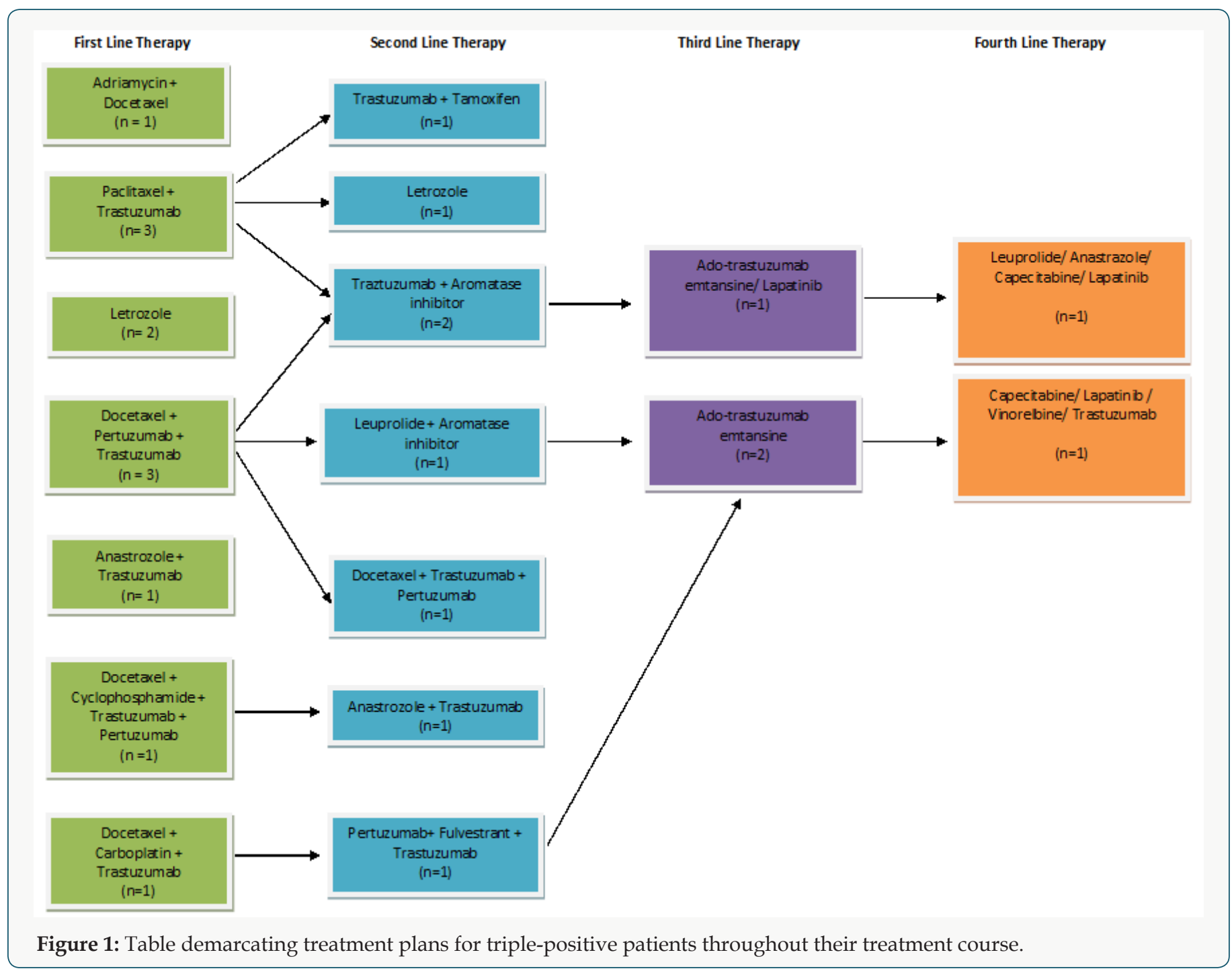

\section{Conclusion}

The 15 patients analyzed at our center with metastatic triple positive breast cancer were treated heterogeneously. It can be stated that the two patients who received single agent therapy performed poorly in comparison to the patients who received combination regimens. All but one patient received trastuzumab in the second line of chemotherapy, and trastuzumab in addition to an aromatase inhibitor was a prefer option in the second line of therapy.

\section{References}

1. Siegel RL, Miller KD, Jemal (2017) A Cancer statistics, 2017. CA: A Cancer Journal for Clinicians 67 (1): 7-30.

2. Metastatic Breast Cancer Network (2017) Metastatic Breast Cancer Incidence and Rates.

3. (2018) American Joint Committee on Cancer, Cancer Staging.

4. Onitilo AA, Engel JM, Greenlee RT, Mukesh BN (2009) Breast Cancer Subtypes Based on ER/PR and Her2 Expression: Comparison of Clinicopathologic Features and Survival. Clin Med Res 7(1-2): 4-13.
5. Sambi M, Haq S, Samuel V (2017) Alternative therapies for metastatic breast cancer: Multimodal approach targeting tumor cell heterogeneity. Breast Cancer: Targets and Therapy 9: 85-93.

6. Advani P, Cornell L, Chumsri S (2015) Dual HER2 blockade in the neoadjuvant and adjuvant treatment of HER2-positive breast cancer. Breast Cancer: Targets and Therapy 7: 321-325.

7. Byunghee Yoo, Amol Kavishwar, Ping Wang (2017) Therapy targeted to the metastatic niche is effective in a model of stage IV breast cancer. Scientific Reports 7: 45060.

8. Johnson S, Roberto H, Im S (2017) Phase III, Randomized Study of Dual Human Epidermal Growth Factor Receptor 2 (HER2) Blockade with Lapatinib Plus Trastuzumab in Combination With an Aromatase Inhibitor in Postmenopausal Women With HER2-Positive, Hormone Receptor-Positive Metastatic Breast Cancer: ALTERNATIVE. Journal of Clinical Oncology 36(8): 741-748.

9. (2017) National Comprehensive Cancer Network, NCCN Guidelines Version 2.

10. Hernandez Aya LF, Ma CX (2016) Chemotherapy principles of managing stage IV breast cancer in the United States. Chinese Clinical Oncology 5(3): 42. 
(c) This work is licensed under Creative

To Submit Your Article Click Here: Submit Article

DOI: $10.32474 /$ OAJOM.2018.02.000127

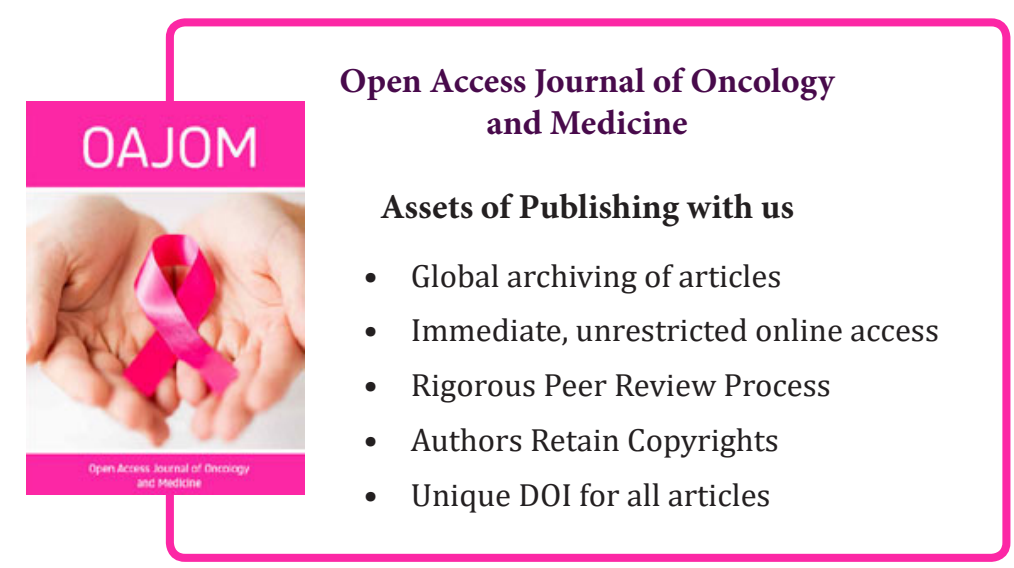

